\title{
McCormick, John P. (2018): Reading Machiavelli. Scandalous Books, Suspect Engagements, and the Virtue of Populist Politics
}

\author{
Princeton: Princeton University Press. 288 Seiten. \$29,95
}

\author{
Dagmar Comtesse
}

Angenommen: 29. Juni 2021 / Online publiziert: 19. Juli 2021

(C) Der/die Autor(en) 2021

Der Kurztitel der erneuten Intervention John McCormicks für eine demokratischpopulistische Lesart Machiavellis trifft gleichzeitig das Innovative wie das Bedeutende dieser Zusammenstellung von überarbeiteten Artikeln: Es geht McCormick um die Art des Lesens, also um eine methodische Frage. Dass McCormick dabei die in „Machiavellian Democracy“ (2011) entwickelte Interpretation Machiavellis verteidigt und weiterhin das politische Ziel einer institutionalisierten ,elite accountability“ (S. 212) verfolgt, ist selbstverständlich. Insofern erhält man mit der Lektüre von „Reading Machiavelli“ auch die kondensierte Fassung von McCormicks Argumentation, Machiavellis Werk als ,consistent advocay for a new form of muscular, populist politics“ (S. 2) zu verstehen. Die Stärke wie auch die Schwäche der Monografie liegen jedoch in der methodischen Reflexion und ihrer praktischen Umsetzung. Stark und deswegen unbedingt lesenswert ist der erste Teil des Buches, der mittels der spezifischen Darstellung der drei Hauptwerke Machiavellis - der „Fürst“, die „Discorsi“ und die „Geschichte von Florenz“ - herausarbeitet, wie Machiavelli die Rolle der Masse aufwertet. Dieses Wie fasst McCormick begrifflich mit „effectual truth of politics“ (S. 3) zusammen, womit eine Art rhetorisch umgesetztes Wirksamkeitskalkül gemeint ist, wie Politik unter der naturhaften Bedingung des Klassenkampfes gelingen kann.

Die Schwäche des Buches liegt im zweiten Teil des Buches, insbesondere in der Rousseau-Interpretation, da McCormick seine im ersten Teil herausgearbeiteten und angewendeten Kriterien der politischen Ideengeschichtsschreibung ignoriert. Während die im zweiten Teil vorgestellten und kritisierten Machiavelli-Interpretationen von Leo Strauss und der Cambridge School nicht unbedingt selbst als Gegenstand der politischen Ideengeschichtsschreibung behandelt werden müssen, hätte McCor-

Dagmar Comtesse $(\bowtie)$

Philosophisches Seminar, WWU Münster, Münster, Deutschland

E-Mail: d.comtesse@uni-muenster.de 
mick unbedingt Rousseau wie Machiavelli lesen müssen. Insofern diskreditiert der Rousseau-Beitrag genau das, was „Reading Machiavelli“ extrem lesenswert macht, nämlich eine die genauen historischen Bedingungen der Theorieproduktion machtanalytisch wahrnehmende politische Ideengeschichtsschreibung.

Insgesamt lassen sich fünf Kriterien bzw. methodische Praktiken der politischen Ideengeschichtsschreibung in den ersten drei Kapiteln ausmachen: a) die Einbeziehung des Gesamtwerks in der hermeneutischen Arbeit am Einzeltext; b) die belegbare Bestimmung der politischen Position des Autors; c) die konkrete Adressierung des Textes; d) der machtanalytisch zu bewertende Einsatz des Textes im zeitgenössischen Diskurs; e) die Einsetzbarkeit des Textes im gegenwärtigen akademischen oder öffentlichen Diskurs. So zitiert McCormick bezüglich der politischen Position Machiavellis im ersten Kapitel dessen Selbstgleichsetzung mit den Massen (S. 23) und argumentiert dafür, dass Machiavelli die ,populare“ Bewertung des Cesare Borgia gleichzeitig übernehme, legitimiere und sich dafür der zeitgenössischen biblischen Symbolik bediene (S. 25-31). Damit adressiere Machiavelli jene mächtigen Leser, die ihre „oder“ die staatliche Herrschaft erfolgreich verankern wollten (S. 32-33). Der Einsatz im zeitgenössischen Diskurs ist das Hauptkriterium für die populistische Lesart der „Discorsi“ im zweiten Kapitel, wird aber auch dort mit der Adressierungsfrage verbunden. Statt die Abschaffung der römischen Republik mit einer Abschwächung politisch-moralischer Tugenden zu erklären, wie es in der republikanischen Tradition üblich sei (S. 46), mache Machiavelli die Zunahme der ökonomischen Ungleichheit in Rom für den Untergang der Republik verantwortlich und bringe die Agrarreform der Gracchen mit dem Aufstand der Wollarbeiter in Florenz, dem Ciompi-Aufstand von 1378, in Verbindung. So wie die Gracchen auf das ökonomische Leid der Mehrheit reagierten, so sei auch der Ciompi-Aufstand als Reaktion auf zunehmende ökonomische Ungleichheit zu sehen und deswegen könne Machiavellis Ratschlag, ,well-ordered republics must keep the public rich and citizen poor“ (S. 57), von der Leserschaft der „Discorsi“, den ,humanist ottimati“ (S. 56), als Warnung verstanden werden. Das Kriterium der Einbettung in das Gesamtwerk wird von McCormick hauptsächlich im dritten Kapitel über die „Geschichte von Florenz“ bemüht, da er die im „Fürst“ und in den „Discorsi“ etablierten Sichtweisen auf die populistische Natur der Politikvorschläge Machiavellis zu ,authoritative guides“ (S. 104) für die Interpretation der „Geschichte von Florenz" macht. Damit richtet sich McCormick gegen die vorherrschende Lesart, die „Geschichte von Florenz“ als „,conservative turn“ (S. 69) Machiavellis zu verstehen. Der Vorgang der Argumentation legt den Schwerpunkt auf eine intensive hermeneutische Arbeit, in der die Beschreibung der Vorgänge in Florenz mit ihrer Bewertung konfrontiert wird (S. 70). So zeige die Beschreibung des Verhaltens der Wollarbeiter während des Ciompi-Aufstands, dass sie nicht nur gerechtfertigte, sondern auch gemäßigte Forderungen stellten, was ihm Widerspruch zu Machiavellis expliziten, abwertenden Äußerungen des Aufstands stehe (S. 82-93). Der Widerspruch wird von McCormick durch das Adressierungskriterium aufgelöst: Da der Text für die Medici produziert worden sei, habe Machiavelli die Doppelrolle inne, Autor einer bestimmten politischen Position und potenzieller Ratgeber der Medici zu sein (S. 102-103). Solche Konstellationen erforderten insbesondere, Machiavelli mit „requisite care“ (S. 104) zu lesen. In allen drei Textvorstellungen nimmt McCormick Bezug auf de- 
ren Einsetzbarkeit im heutigen öffentlichen Diskurs. Besonders im Vergleich zu den Interpretationen von Strauss und der Cambridge School macht McCormick deutlich, dass Machiavelli gegen jenen „,spirit“ in Stellung gebracht werden könnte, der „overly alarmist responses to ,populism“ and a persistently expressed mistrust of majoritarianism“ (S. 205) in den Diskurs einbringt.

Im zweiten Teil werden die Machiavelli-Interpretationen und Interpretationsweisen von Strauss und der Cambridge School mit McCormicks eigener Lesart konfrontiert und insbesondere durch den Verweis auf die klassenspezifischen Institutionen und Politiken in den Machiavelli-Texten plausibel als antipopulistische Lesarten vorgestellt. Der Rousseau-Beitrag analysiert die Funktion der Darstellung der römischen Republik im „Contrat social“ und ist gerade vor dem Hintergrund der von McCormick selbst auf die Machiavelli-Texte angewendeten Kriterien der politischen Ideengeschichtsschreibung nicht überzeugend. Es wäre sehr wünschenswert, diese Kriterien auf Rousseau anzuwenden, nicht nur um McCormicks These zu widerlegen, dass Rousseaus Rom-Darstellung ,deliberately undermines Machiavelli's efforts to reconstruct and promote institutions that both maximize the participation of poor citizens in popular governments and facilitate their efforts to control or contain economic and political elites“ (S. 109). Insbesondere der Beleg der politischen Position Rousseaus in den „Briefen aus den Bergen“ würde zeigen, dass Rousseau konkret im Kampf gegen die Genfer Oligarchie engagiert war und zudem den engen Bürgerbegriff der alten Bürgerschaft Genfs ablehnte und für eine Inklusion aller Männer Genfs plädierte (7. Brief). Dies ginge natürlich einher mit der Adressierungsfrage, welche für den „Contrat social“ ganz anders zu beantworten ist als für die „Briefe vom Berge“ oder die Verfassungsentwürfe. Schließlich wäre mit Blick auf den zeitgenössischen Diskurs ganz einfach festzustellen, wie sehr Rousseau, der ab 1762 zu den politisch Verfolgten Europas gehörte, für seine Kritik am Künstlerund Gelehrtenestablishment und seinen Einsatz für die moralische, politische und soziale Gleichheit aller Männer beispielsweise in vom Versailler Hof finanzierten Theaterstück „Les philosophes“ als Vierbeiner diffamiert wurde. Der Blick auf den zeitgenössischen Diskurs würde auch zeigen, dass die Idee der Volkssouveränität zwar von den Radikalen wie Diderot und d'Holbach geteilt wurde, aber kein anderer die Institutionen entwarf, welche die Volkssouveränität als Selbstgesetzgebung sichern sollten. Die intermediäre Funktion der Volkstribune in Rousseaus Rom-Darstellung würde mit Blick auf das Gesamtwerk, was im Übrigen Valentina Arena (2016) getan hat, nur zeigen, dass Rousseau die Trennung von Legislative und Exekutive überaus ernst nimmt und nur im Fall der korsischen Verfassung im Sinne einer lokalen Selbstverwaltung aufgibt. Mit anderen Worten: „Reading Rousseau“ like „Reading Machiavelli“ wäre ein Gewinn.

Funding Open Access funding enabled and organized by Projekt DEAL.

Open Access Dieser Artikel wird unter der Creative Commons Namensnennung 4.0 International Lizenz veröffentlicht, welche die Nutzung, Vervielfältigung, Bearbeitung, Verbreitung und Wiedergabe in jeglichem Medium und Format erlaubt, sofern Sie den/die ursprünglichen Autor(en) und die Quelle ordnungsgemäß nennen, einen Link zur Creative Commons Lizenz beifügen und angeben, ob Änderungen vorgenommen wurden. 
Die in diesem Artikel enthaltenen Bilder und sonstiges Drittmaterial unterliegen ebenfalls der genannten Creative Commons Lizenz, sofern sich aus der Abbildungslegende nichts anderes ergibt. Sofern das betreffende Material nicht unter der genannten Creative Commons Lizenz steht und die betreffende Handlung nicht nach gesetzlichen Vorschriften erlaubt ist, ist für die oben aufgeführten Weiterverwendungen des Materials die Einwilligung des jeweiligen Rechteinhabers einzuholen.

Weitere Details zur Lizenz entnehmen Sie bitte der Lizenzinformation auf http://creativecommons.org/ licenses/by/4.0/deed.de. 